Research papers

\title{
Unsaturated hydraulic properties of heterogeneously packed sands: A pore- scale computational study
}

\author{
Mojtaba G. Mahmoodlu ${ }^{\mathrm{a}, \mathrm{b}, *}$, Martinus Th. van Genuchten ${ }^{\mathrm{b}, \mathrm{c}}$, Thomas Sweijen ${ }^{\mathrm{b}}$, Amir Raoof $^{\mathrm{b}}$ \\ a Department of Watershed and Rangeland Management, Gonbad Kavous University, Gonbad Kavous, Iran \\ b Department of Earth Sciences, Utrecht University, Utrecht, The Netherlands \\ ${ }^{\mathrm{c}}$ Center for Environmental Studies, CEA, São Paulo University, Rio Claro, SP, Brazil
}

\section{A R T I C L E I N F O}

This manuscript was handled by C. Corradini, Editor-in-Chief, with the assistance of Corrado Corradini, Associate Editor

Keywords:

Soil water retention curve (SWRC)

Intrinsic permeability

Heterogeneous sands

Discrete element method

Pore structure

\begin{abstract}
A B S T R A C T
The macroscopic effects of soil textural heterogeneity and fine-scale soil layering on unsaturated flow remain poorly understood. In this study we used the Discrete Element Method (DEM) to numerically generate artificial particle packings of heterogeneous sands from which the pore structure can be extracted. Packings were generated from known grain size distributions following sand grain mixing and fine-scale profile layering. Five sands with different mean grain sizes were used to investigate the effects of heterogeneity on their pore structure, including changes in the permeability and soil water retention (SWR). Heterogeneous media were created by mixing a relatively fine sand with coarser sands. In addition, we created layered media having sharp as well as transitional interfaces between the two sand samples. Mixing fine and coarse sand caused reductions in the average pore body and pore throat sizes of the coarser sands, and hence also in their intrinsic permeability. The layered media with a transitional interface showed lower porosities at the interface because of penetration of small particles into the larger pores, while the porosities increased at sharp interfaces. The nonlinear relationships between permeability and the average pore body and throat radii were explored using different unimodal sands. Mixing fine and coarse sand caused a decrease in the capillary pressure at a given water content of the new medium, and hence larger values of the van Genuchten $\alpha$ parameter. The SWRCs of the layered soils with a sharp interface were best described using bimodal functions. Sharp interfaces caused a non-monotonic change in the drainage curve due to discrepancies between the pore throat radii of the two adjacent sands. This change was influenced by $d_{50}$ of the sands used for the layered media. In contrast to the sharp interface, a monotonic (smooth) change in the drainage curve was observed for two sands having small differences in their $d_{50}$ values. The study gave considerable insight into how particle heterogeneity and layering affects the hydraulic properties of unsaturated media.
\end{abstract}

\section{Introduction}

Reliable estimates of the unsaturated soil hydraulic properties are required for effective management and simulations of many near-surface hydrological processes such as infiltration, runoff, groundwater recharge, irrigation and optimal water use in agricultural operations, and contaminant transport in the vadose zone (Zhu and Sun, 2012; Di Prima et al., 2016). Applications also include such diverse problems as slope stability analyses (Mukhlisin et al., 2011; Antinoro et al., 2017), flow and seepage into and through earth dams (Alonso and Cardoso, 2010; Al-Ismaily et al., 2015) and infiltration into and through waste disposal sites (Lopes et al., 2013).

The hydraulic properties of variably-saturated porous media include the soil water retention curve relating capillary pressure and fluid saturation, and the unsaturated soil hydraulic conductivity function. A large number of experimental protocols have been developed over the years to estimate either one or both of these functions using such methods as hanging water columns or sand boxes, pressure cells, pressure plate extractors, suction tables, soil freezing, falling head methods, infiltrometer and permeameter devices, laboratory one-step and multistep outflow methods, centrifugation and evaporation methods, and other approaches (Dane and Hopmans, 2002; Nimmo et al., 2002; Scanlon et al., 2002; Bittelli and Flury, 2009; Zhuang et al., 2017).

Notwithstanding the plethora of available methods, a major challenge for accurate delineation of field-scale hydraulic properties at both very small and large spatial scales remains the overwhelming heterogeneity of the subsurface (Chen et al., 2012; Hohenbrink and Lischeid,

\footnotetext{
* Corresponding author at: Department of Watershed and Rangeland Management, Gonbad Kavous University, Gonbad Kavous, Iran.

E-mail address: m.gharehmahmoodlu@uu.nl (M.G. Mahmoodlu).
} 
2015; Sakaki and Smits, 2015; Morbidelli et al., 2016). At relatively small scales, heterogeneities are manifested by non-uniform particleand pore-size distributions, soil textural layering and soil structure (Mohanty and Zhu, 2007; Coppola et al., 2009; Huang et al., 2011; Arora, 2012; Sakaki and Smits, 2015). The totality of micropores (defined here as those smaller than $75 \mu \mathrm{m})$ and macropores $(>75 \mu \mathrm{m})$, including their geometries and spatial distributions, are the primary determinants of two-phase fluid flow and contaminant transport in porous media. Fluid flow and capillary displacement processes are strongly affected by pore throat sizes since they provide the constrictions along the flow paths (Perrier et al., 1996; Raoof et al., 2010; Falode and Manuel, 2014; Mahmoodlu et al., 2016). Precise studies of the effects of soil textural heterogeneity and soil layering or stratification on the unsaturated soil hydraulic properties require detailed analyses of the geometry of sand grains and related pore structures in terms of prevailing pore body and pore throat sizes. Quantification of these geometry effects offers an opportunity to relate the soil water retention properties to soil structural components (e.g., Perrier, et al., 1996). Although the particle size distribution is relatively easy to measure using a range of techniques, estimation of grain positions and pore structures cannot be done in the same way (Gee and Or, 2002; Mehlhorn et al., 2008; Mahmoodlu et al., 2016). X-ray microtomography (which uses $\mathrm{x}$-ray radiation to create cross-sections of a physical object) together with image processing (methods to convert an image into digital form and perform some operations on it) can be used to obtain more directly details of pore structure geometries as defined by the size, arrangement, and connectivity of pores (e.g., Peth et al., 2010; Cnudde and Boone, 2013; Vaz et al., 2014). Unfortunately, X-ray microtomography is still relatively expensive (Mehlhorn et al., 2008) and image processing relatively time consuming.

In this study, we used an alternative approach to investigate the effects of small-scale heterogeneities on the soil hydraulic properties. Following Keller et al. (2013) and Mahmoodlu et al. (2016), we considered idealized grain geometries to obtain the pore structures of soils having predefined unimodal and bimodal particle size distributions (having a single peak and two peaks, respectively), as well as of layered soil samples. Particle packings were generated using the discrete element method (DEM), which allows one to simulate the movement of grains during compaction of the particles (Cundall and Strack, 1979). Computations were carried out using the open source Yade-DEM software (Šmilauer et al., 2015) which also allows us to compute the hydraulic properties as well as the soil water retention curve (SWRC). Once a packing of spheres was constructed, the pore space (consisting of pore bodies and pore throats) was extracted using regular triangulation (Chareyre et al., 2012), which leads to an assembly of grainbased tetrahedra. Using this assembly, the intrinsic permeability can be determined (e.g. Tong et al., 2012; Chareyre et al., 2012) as well as the SWRC following approaches by Yuan et al. (2015) and Sweijen et al. (2016).

Specific objectives of our study were to: (1) use the discrete element model to extract pore structures of different heterogeneous particle packings; (2) explore the effects of vertical heterogeneity (i.e., soil textural layering) and heterogeneous sphere packings on the pore structures; and (3) investigate the effects of these heterogeneities on the soil water retention and the intrinsic permeability, including the van Genuchten hydraulic parameters $\alpha$ and $n$.

\section{Sand properties}

As input to the discrete element model (DEM), we used the synthetic particle size distributions of five sands, each one having a relative narrow particle size distribution as shown in Fig. 1. Particle sizes varied from 76 to $1190 \mu \mathrm{m}$ (Table 1). Sand S1, which had the finest texture (Fig. 1a, Table 1), was used to create heterogeneous soil samples by mixing it with the other sands (Fig. 1b), thus allowing us to explore the mixing effects on grain size and the resulting pore size distributions reflecting grain heterogeneity. The coefficients of uniformity $\left(C_{u}\right)$, curvature $\left(C_{c}\right)$, and sorting $\left(S_{o}\right)$ were estimated for all sands and their mixtures as follows (Dubois et al., 2009; Katukiza et al., 2014):

$C u=\frac{d_{60}}{d_{10}}$

$C_{c}=\frac{d_{30}^{2}}{d_{10} \times d_{60}}$

$S_{o}=\sqrt{\frac{d_{75}}{d_{25}}}$

where $d_{x}$ denotes the grain diameter (in $\mathrm{mm}$ ), for which $x \%$ of the grains are finer.

Results of the coefficients of uniformity, curvature, and sorting are given in Table 1 . Because the coefficient of uniformity was less than 4 for the five main sands, the samples were considered to be uniformly graded by containing essentially identical particle sizes (Purushothama, 2007). The coefficient of curvature was used as another useful measure of the shape of the particle size distribution. The sorting coefficient, which also describes the distribution of grain size, was additionally considered as a measure of the uniformity of the soil samples. The data in Table 1 show that the three coefficients $\left(C_{u}, C_{c}\right.$ and $\left.S_{o}\right)$ all indicate similar narrow degrees of sorting of the sands. The degree of sorting of the mixtures declined towards the coarser sands (i.e., S4, S5) because of the creation of increasingly more bimodal grain size distributions.

\section{Pore scale analysis}

In this section we describe how the homogeneous and heterogenous sand media were constructed and analyzed in terms of their hydraulic properties using the discrete element method. We first provide details of the packing procedures and resulting pore structures as defined by the size, arrangement and connectivity of the pores. We next investigate the pore body radius distributions of the main sands, followed by a study of heterogeneous sphere packings obtained by mixing fine sand S1 with the other main sands (samples S2 to S5 in Table 1), as well as of the effects of soil textural layering of the sand packings. The discrete element method is subsequently used to evaluate the intrinsic permeability and soil water retention properties of the different sand packings (i.e., the single, mixed and layered media).

\subsection{Sand packings}

To limit errors in the simulations while minimizing simulation times, we decided to use for our calculations 2000 particles from a given particle size distribution. In view of our interest to investigate the implications of both grain size heterogeneity and soil layering, we considered two different ways of spherical particle packings. For the individual sand samples (five sand samples, i.e., S1, S2, S3, S4 and S5) and the mixtures of two sands (resulting in four sand samples; S1\&S2, S1\&S3, S1\&S4 and S1\&S5), particles were inserted in a large box such that clouds of particles were generated (following procedures by Mahmoodlu et al. 2016).

We assumed normal elasticity, tangential elasticity, and sliding as the main processes taking place at the contact points of spheres. Normal elasticity was calculated using a linear contact law (Belheine et al., 2009; Mahmoodlu et al., 2016). When two particles are in contact and pushed toward each other by the surrounding medium, they are flattened at their contact point. The tendency of both particles to keep their initial shapes may cause an elastic force $f_{\mathrm{n}}$ :

$f_{n}=k_{n} \delta_{n}$

where $k_{n}$ is the stiffness of the particles and $\delta_{n}$ denotes the normal displacement.

A tangential force, $f_{t}$, may arise as a result of shear at the contact 

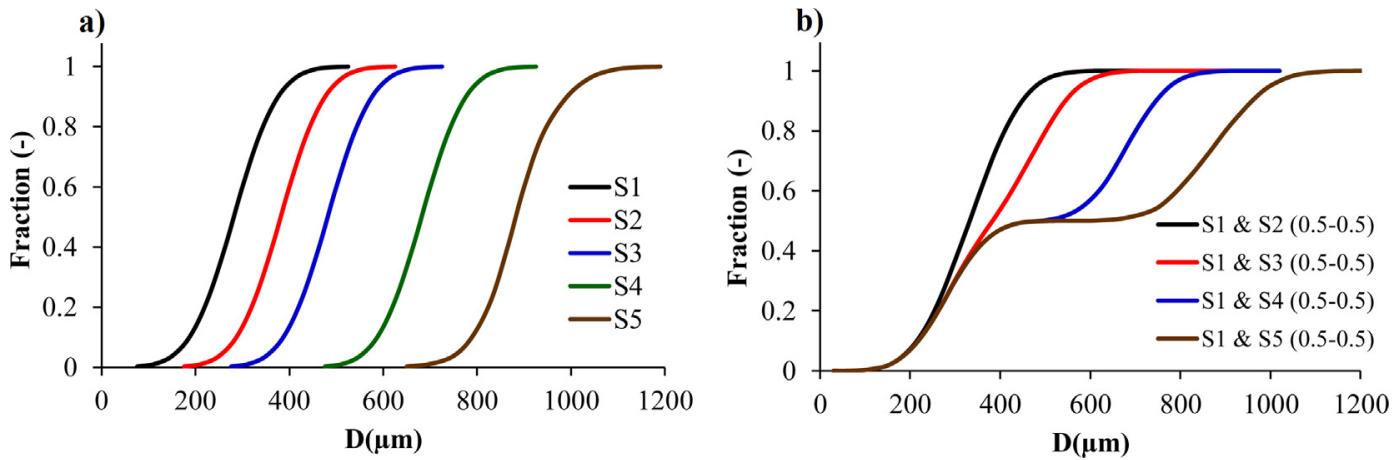

Fig. 1. Synthetic particle size distributions of the five main sands (a) and their mixtures (b).

point. When the tangential force reaches a certain threshold value, the contact point will break and cause sliding of the two particles. This process is described by

$f_{t} \leqslant f_{n} \tan \phi$

where $\phi$ denotes the friction angle used to estimate the threshold value. For each particle, a force balance is made by including forces due to contacts with other particles, the boundary conditions, and the effects of gravity. The resulting net force gives the acceleration of the particle using Newton's second law (Cundall and Strack, 1979; Belheine et al., 2009).

The grain-scale parameters needed for this approach were taken from previous DEM studies. However, mechanical parameterization of the particles could be done arbitrarily since the porosity was used as a proxy for the state-of-stress. Thus, a confining stress was applied such that a particular porosity was obtained. Values of the particle stiffness, friction angle, and Poisson ratio were fixed at $9.6 \times 108 \mathrm{~Pa}, 30^{\circ}$, and 0.04, respectively (Belheine et al., 2009). For our simulations we allowed the six surrounding boundaries of the domain to confine and drag the particles until a porosity value of 0.45 was reached. After this point, only the top boundary was allowed to move down to further confine the particle packings until a target porosity of 0.35 resulted.

For the sand packings consisting of two layers, different interfaces between the two sand layers were created during packing. For these cases we first inserted a number of particles into a large box such that a cloud of particles was generated for the first (bottom) layer, similarly as for the previous (mixed) samples. But now five of the boundaries of the domain were kept constant, while the top boundary was allowed to move down to confine the packings until the target porosity of 0.35 was reached (the average porosity of the coarse sand). Once reached, we froze the bottom layer and then started packing the top layer in the same way as the first layer. The resulting interface between the two layers was very much dependent upon the grain diameter (or $d_{50}$ ) of the first layer. If we used a relatively fine sand as the first layer (Fig. 2a, c), and a coarser sand as the top layer (i.e., $d_{50}$ of sand $S 1$ being less than $d_{50}$ of sand S2), a very sharp interface resulted (sand samples S1-S2, S1S3, S1-S4 and S1-S5 presented in Table 2). On the other hand, using a coarser sand at the bottom and finer sand $\mathrm{S} 1$ at the top produced a much smoother interface because of the intrusion by gravity of some smaller particles from the top layer into the coarser pores of the bottom layer (Fig. 2b, d and sand samples S2-S1, S3-S1, S4-S1 and S5-S1 presented in Table 2). To keep the length of both layers in the sand packings with two layers (i.e., those having an interface) constant, the mass fraction of Sand S1 in the sand packings made up of two layers was variable among the different samples (approximately 70\%, 80\%, $90 \%$, and $95 \%$ for the packings of S1-S2, S1-S3, S1-S4, and S1-S5, respectively). For instance, the packing of S1-S5 required in this way only $5 \%$ of S5. Hence, large fractions of S1 in all sand packings consisting of two layers produced very minor discrepancies between the pore radii and/or pore throat distributions of the S1-S2 and S1-S5 sand packings. Compaction was accounted for in the pore-scale calculations by repeated repacking and rearrangement of the spherical particles to decrease porosities to the prescribed values, with concomitant changes in the pore structures.

\subsection{Pore structure}

A regular triangulation method was used to extract the geometry of the various pore structures (Chareyre et al., 2012). The method proceeds by dividing the pore space into tetrahedrons, with each tetrahedron serving as a pore unit. Each tetrahedron is then surrounding one pore body and has four vertices that are located at the centers of the spherical particles (Fig. 3). The sides, or facets, of the tetrahedrons are the locations at which two pore bodies are connected. The facet is the narrowest transect between two connecting pore units and is referred to as the pore throat.

Although several studies suggest a distribution in pore connectivity values due to the natural structure of porous media (e.g., Raoof et al., 2010; Vasilyev et al., 2012), our approach assumes that four pore throats are connected to each pore body. Each pore throat in this way is

Table 1

Selected properties of the sands used in this study.

\begin{tabular}{|c|c|c|c|c|c|c|c|c|c|c|c|}
\hline Sample & Minimum & Maximum & $d_{10}$ & $d_{25}$ & $d_{30}$ & $d_{50}$ & $d_{60}$ & $d_{75}$ & $\mathrm{C}_{\mathrm{u}}$ & $\mathrm{C}_{\mathrm{c}}$ & $\mathrm{S}_{\mathrm{o}}$ \\
\hline \multicolumn{12}{|l|}{$\mu \mathrm{m}$} \\
\hline S1 & 76 & 525 & 188 & 230 & 245 & 283 & 300 & 330 & 1.60 & 1.06 & 1.20 \\
\hline S2 & 176 & 625 & 290 & 330 & 345 & 385 & 400 & 435 & 1.38 & 1.03 & 1.15 \\
\hline S3 & 276 & 725 & 388 & 430 & 445 & 485 & 500 & 530 & 1.29 & 1.02 & 1.11 \\
\hline S4 & 476 & 925 & 588 & 630 & 645 & 685 & 700 & 730 & 1.19 & 1.01 & 1.08 \\
\hline S5 & 650 & 1190 & 790 & 835 & 845 & 885 & 900 & 932 & 1.14 & 1.00 & 1.06 \\
\hline S1\&S2 & 76 & 625 & 239 & 280 & 295 & 334 & 350 & 382.5 & 1.46 & 1.04 & 1.17 \\
\hline S1\&S3 & 76 & 725 & 288 & 330 & 345 & 384 & 400 & 430 & 1.39 & 1.03 & 1.14 \\
\hline S1\&S4 & 76 & 925 & 388 & 430 & 445 & 484 & 500 & 530 & 1.29 & 1.02 & 1.11 \\
\hline S1\&S5 & 76 & 1190 & 489 & 532.5 & 545 & 584 & 600 & 631 & 1.23 & 1.01 & 1.09 \\
\hline
\end{tabular}

$C_{u}$ : Uniformity coefficient; $C_{c}$ : Curvature coefficient; $S_{o}$ : Sorting coefficient. 

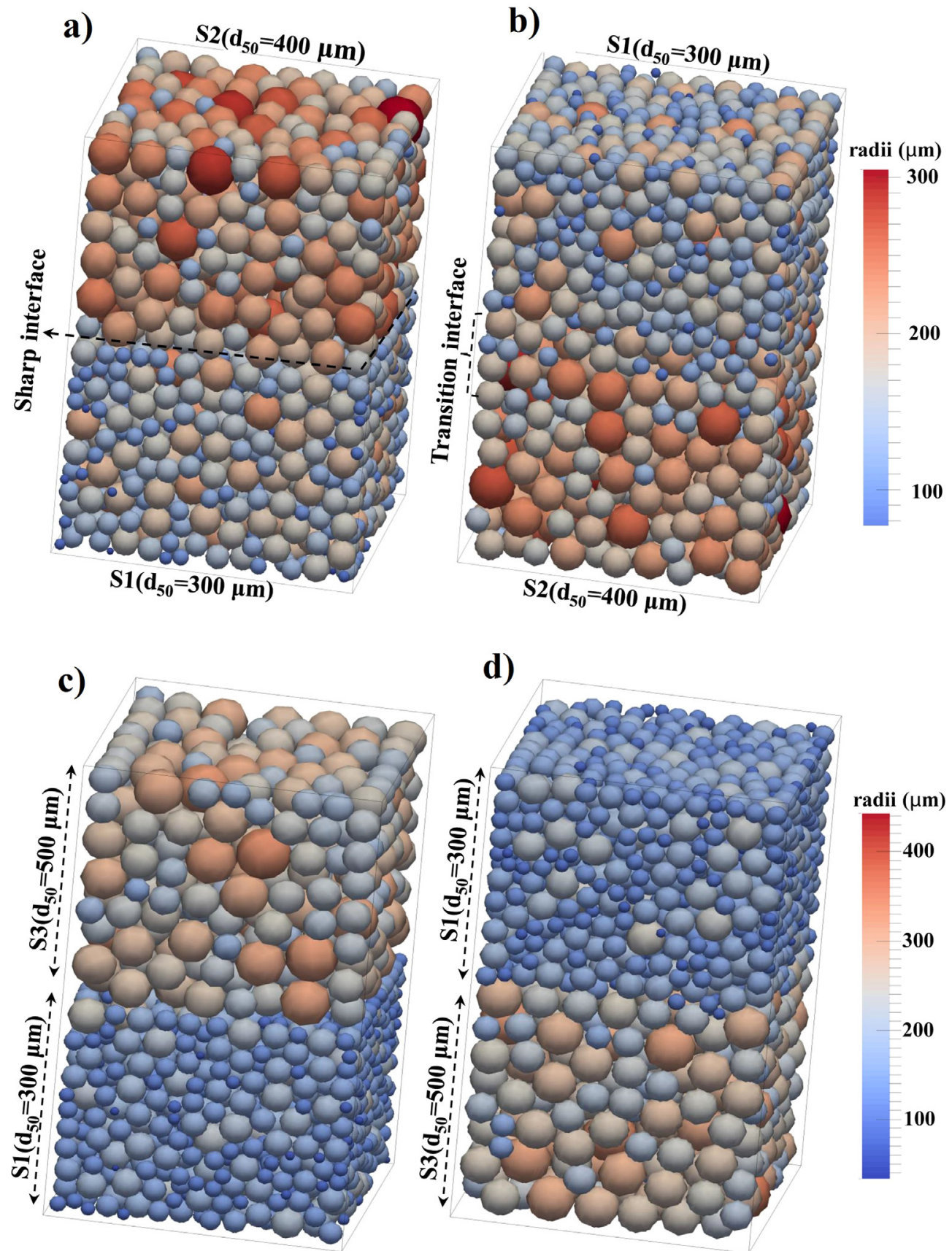

d)

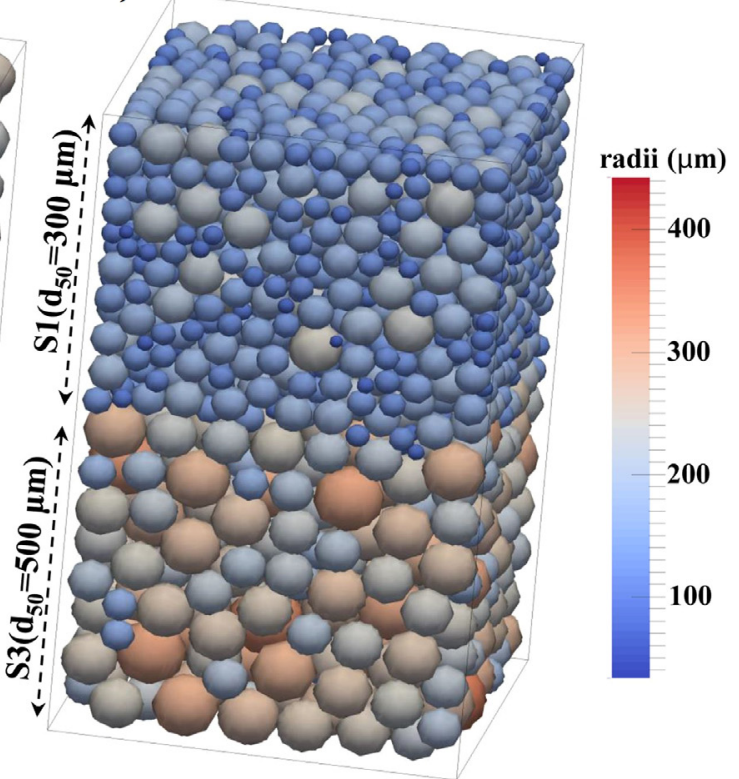

Fig. 2. Sphere packings of two layers with different mean particle diameters $\left(d_{50}\right)$ with $d_{50(\mathrm{~S} 1)}<d_{50(\mathrm{~S} 2)}<d_{50(\mathrm{~S} 3)}$. a): a sharp interface with $\mathrm{S} 1$ at the bottom and $\mathrm{S} 2$ at the top, b) a transitional interface with S2 at bottom and S1 at the top, c) a sharp interface with S1 at bottom and S3 at the top, and d) a transitional interface with S3 at the bottom and S1 at the top.

then surrounded by three particles to which an inscribed circle can be fitted (Fig. 3). The radius of this circle, $R_{i j}$, is taken as the pore throat radius (Torskaya et al., 2014). Inside a pore unit, an inscribed sphere exists that touches the four surrounding particles (e.g. Sweijen et al., 2016). The approach using inscribed spheres is often referred to as the pore finite volume method (Gladkikh and Bryant, 2005; Unsal et al., 2009; Mousavi and Bryant, 2012; Prodanović et al., 2015), while the corresponding radius is denoted as $R_{i}$.

\subsection{Calculation of the permeability}

Following a study by Chareyre et al. (2012), the pore finite volume method as implemented in the DEM was used to compute the permeability of granular materials in the sphere packings. This method allows one to solve for the pressure distribution under fully water saturated conditions. The approach assumes that one pressure value can be associated with each pore unit. The flux, $q_{i j}\left[\mathrm{~L}^{3} \mathrm{~T}^{-1}\right]$, of water from a given pore unit $i$ to pore unit $j$ is then given by (Vasilyev et al., 2012):

$q_{i j}=k_{i j} \frac{\left(p_{i}-p_{j}\right)}{l_{i j}}$

where $p_{i}$ and $p_{j}\left[\mathrm{ML}^{-1} \mathrm{~T}^{-2}\right]$ are the pressures in pore unit $i$, and $j$, respectively, $l_{i j}[\mathrm{~L}]$ is the length between the centers of pore units $i$ and $j$, and $k_{i j}\left[\mathrm{M}^{-1} \mathrm{~L}^{5} \mathrm{~T}\right]$ denotes the hydraulic conductivity of the facet (pore throat) between pores $i$ and $j$. The latter is given by: 
Table 2

van Genuchten soil hydraulic parameters of the various samples.

\begin{tabular}{|c|c|c|c|c|c|c|c|c|}
\hline Sample & $\theta_{r}\left(\mathrm{~m}^{3} \mathrm{~m}^{-3}\right)$ & $\theta_{s}\left(\mathrm{~m}^{3} \mathrm{~m}^{-3}\right)$ & $w_{1}(-)$ & $\alpha_{1}\left(\mathrm{kPa}^{-1}\right)$ & $\alpha_{2}\left(\mathrm{kPa}^{-1}\right)$ & $n_{1}(-)$ & $n_{2}(-)$ & Description \\
\hline S1 & 0.060 & 0.350 & 1.00 & 0.141 & - & 15.3 & - & individual \\
\hline $\mathrm{S} 2$ & 0.076 & 0.350 & 1.00 & 0.189 & - & 14.9 & - & individual \\
\hline S3 & 0.063 & 0.350 & 1.00 & 0.231 & - & 16.1 & - & individual \\
\hline S4 & 0.063 & 0.350 & 1.00 & 0.302 & - & 19.1 & - & individual \\
\hline S5 & 0.062 & 0.350 & 1.00 & 0.389 & - & 29.5 & - & individual \\
\hline S1\&S2 & 0.064 & 0.350 & 1.00 & 0.162 & - & 20.0 & - & mixed (0.5-0.5) \\
\hline S1\&S3 & 0.063 & 0.350 & 1.00 & 0.198 & - & 18.4 & - & mixed (0.5-0.5) \\
\hline S1\&S4 & 0.072 & 0.350 & 1.00 & 0.280 & - & 21.4 & - & mixed (0.5-0.5) \\
\hline S1\&S5 & 0.062 & 0.350 & 1.00 & 0.359 & - & 18.2 & - & mixed (0.5-0.5) \\
\hline S1-S2 & 0.072 & 0.350 & 1.00 & 0.168 & - & 10.0 & - & sharp interface \\
\hline S1-S3 & 0.065 & 0.350 & 0.50 & 0.240 & 0.141 & 18.6 & 11.1 & sharp interface \\
\hline S1-S4 & 0.062 & 0.350 & 0.50 & 0.326 & 0.143 & 11.8 & 11.8 & sharp interface \\
\hline S1-S5 & 0.052 & 0.350 & 0.50 & 0.428 & 0.144 & 22.3 & 9.9 & sharp interface \\
\hline S2-S1 & 0.172 & 0.350 & 1.00 & 0.171 & - & 68. & - & transition interface \\
\hline S3-S1 & 0.200 & 0.350 & 1.00 & 0.197 & - & 384. & - & transition interface \\
\hline S4-S1 & 0.205 & 0.350 & 1.00 & 0.232 & - & 347. & - & transition interface \\
\hline S5-S1 & 0.223 & 0.350 & 1.00 & 0.272 & & 371. & & transition interface \\
\hline
\end{tabular}

$k_{i j}=\frac{A_{i j}^{f} R_{i j}^{2}}{2 \mu}$

where $A_{i j}^{f}\left[\mathrm{~L}^{2}\right]$ is the smallest transect of the pore throat, $\mu\left[\mathrm{ML}^{-1}\right]$ is the dynamic viscosity, and $R_{i j}[\mathrm{~L}]$ is the hydraulic radius of the facet, i.e.,

$R_{i j}=\frac{\Theta_{i j}}{\omega_{i j}}$

in which $\Theta_{i j}\left[\mathrm{~L}^{3}\right]$ is the pore throat volume, and $\omega_{i j}\left[\mathrm{~L}^{2}\right]$ the surface of the pore-throat. We refer readers to Chareyre et al. (2012) for more details. Under steady-state conditions, and assuming incompressible flow, we further use the continuity equation given by (Zhang et al., 2015):

$\sum_{j=1}^{4} q_{i j}=0$

Eqs. (6), (7) and (9) were solved using an implicit numerical scheme to obtain pressure values (Raoof et al., 2010). The total flux through the medium was subsequently used to compute the permeability of the medium.

\subsection{Capillary pressure-saturation calculations}

The capillary pressure-saturation (or soil water retention, SWRC) curves were estimated using a module inside Yade-DEM that was implemented by Yuan et al. (2015) and Sweijen et al. (2016). We assumed that the top boundary of the simulated domain was an air reservoir with pressure $P_{a i r}$, and the bottom boundary a water reservoir at a fixed pressure, $P_{w}$. The capillary pressure, $P_{c}$, was set equal to the pressure difference between the two reservoirs, which is valid for quasi-static conditions:

$P_{c}=P_{\text {air }}-P_{w}$

To estimate the capillary pressure - saturation curve for drainage, the non-wetting air pressure was increased in steps to allow air to move into increasingly smaller pore throats. Air will enter a water-saturated pore unit if the capillary pressure is larger than the entry pressure of the pore throat $\left(P_{e}\right)$, which then can be obtained using the Young-Laplace equation:

$P_{e}=\frac{2 \gamma}{R_{i j}}$

where $\gamma$ is the surface tension and $R_{i j}$ the radius of the inscribed circle in the pore throat (Fig. 3). Following a study by Zhang et al. (2015), the capillary pressure during imbibition must be lower than the entry pressure before water will invade an air-saturated pore unit. At each pressure step, the equilibrium positions of the air-water interfaces within the network were determined. This information was then used to calculate the average saturation of the network at any given capillary pressure (Raoof and Hassanizadeh, 2012).

To estimate the complete SWRC, the simulation procedure was as follows (Sweijen et al., 2016 and 2017; Yuan et al., 2015): After first selecting a capillary pressure value, we determined if air could invade a water-saturated pore body from a neighboring air-invaded pore body. For invasion to occur, both pore bodies had to be connected to a corresponding reservoir. Disconnected (e.g. trapped) water-saturated pores were used estimate residual water saturations. An algorithm was used next to keep track of disconnected air and water regions to ensure that no displacement between the disconnected pores could occur. However, in real porous media with hydrophilic surfaces, water may also be stored in edges and corners as well as in in the form of adsorbed films on the grains. a)

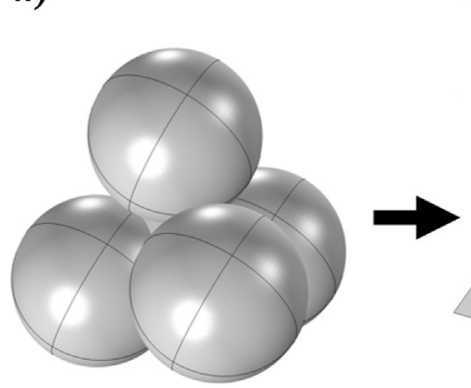

b)

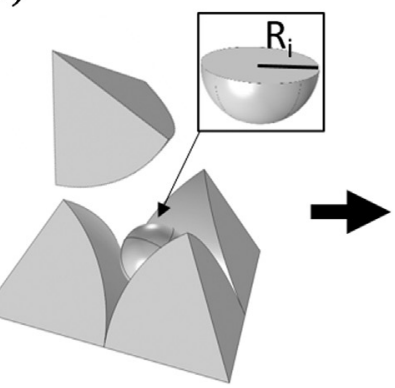

c)

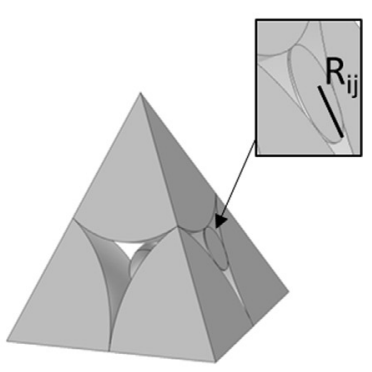

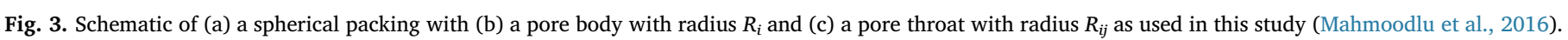


Once the complete SWRCs were obtained, we analyzed the curves in terms of the standard unimodal van Genuchten function (1980) given by

$\theta^{w}\left(p_{c}\right)=\theta_{r}^{w}+\frac{\theta_{s}^{w}-\theta_{r}^{w}}{\left[1+\left(\alpha p_{c}\right)^{n}\right]^{1-1 / n}} \quad p_{c}<0$

where $\theta^{w}$ is the volumetric water content, $\theta_{r}^{w}$ denotes the residual water content, $\theta_{s}^{w}$ is the saturated water content (assumed in this study to be equal to the porosity, $\phi$ ), $\alpha$ and $n$ are semi-empirical shape parameters (further referred to as van Genuchten parameters), and $P_{c}$ denotes capillary pressure. Eq. (12) was fitted to the SWRC data using the RETC software (van Genuchten et al., 1991), which is a nonlinear least-squares optimization program commonly used to for analyzing observed unsaturated soil hydraulic data.

The SWRC in some cases, especially for the layered samples, could be described better using a bimodal retention function. For those cases we used the bimodal formulation introduced by Durner (1994):

$S_{e}{ }^{w}=\sum_{i=1}^{k} w_{i}\left[\frac{1}{1+\left(\alpha_{i} p_{c}\right)^{n_{i}}}\right]$

where $k$ is the number of subsystems that form the total pore-size distribution (in this study, $k=2$ ) and $w_{i}$ are weighting factors for the sub-curves, subject to $0<w_{i}$ less than 1 and $w_{1}+w_{2}=1$. Similarly as for the unimodal curve, the parameters of the two sub-curves of Eq. (13) were subjected to the constraints $\alpha_{i}>0, m_{i}>0, n_{i}>1$.

\section{Results}

\subsection{Effect of heterogeneous packing on pore structures}

Fig. 4a, b shows cumulative frequency distributions of the pore body and pore throat radii for Sands S1 to S5 (the main sands), as well as of

a)

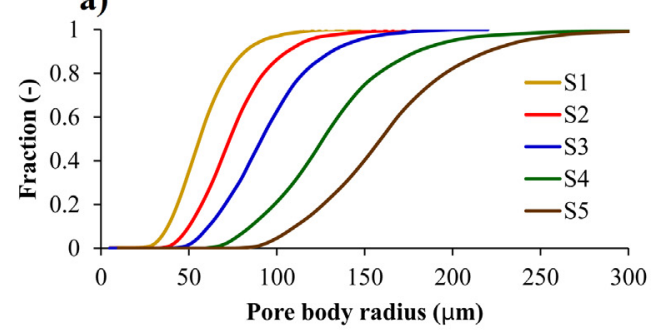

c)

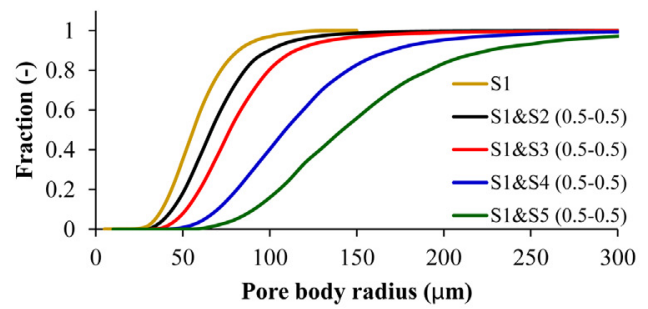

e)

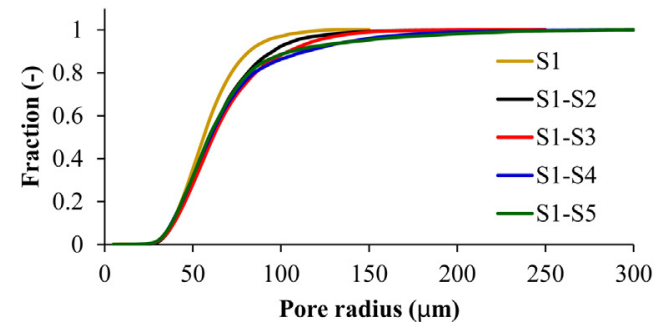

the sand mixtures (Fig. 4c, d) and the two-layered packings (Fig. 4e, f). Results show relatively uniform distributions of the pore body and pore throat radii of the main sands due to fairly uniform increases in the grain diameters (Fig. 4a, b). The plots indicate that particle mixing affected the pore- and throat-size distributions at the given porosity of 0.35 (Fig. 4c, d). Mixing Sand S1 with the coarser sand grains (i.e., Sands S4 and S5) created mixtures with much larger pore body and throat radii as compared to Sand S1 as such. Linear relationships were obtained between the average pore throat and pore body radii during mixing, similarly as in our earlier study on compaction (Mahmoodlu et al., 2016).

Since SWRCs are dominated by pore throat radii, at least in the wet range, one may expect fairly uniform changes in the SWRC of each sand (S1 to S5) when the grain diameters increase, but less uniform changes when considering the mixed media (Mahmoodlu et al., 2016). We obtained a linear relationship between the average pore throat and pore body radii when mixing sand $\mathrm{S} 1$ with the other sands. To explore the effect of particle diameter on pore size during mixing of the sands, the average pore body and pore throat radii of all sands were calculated and then plotted versus $d_{50}$. The overall linear correlation indicates that the average pore body and throat radii can be estimated reasonably well at a given porosity value. These correlations can be useful to generate pore network models of soils with known porosity values. Our calculations showed that the relationship between the average pore body and/or throat radius with $d_{50}$ is nonlinear if the difference between $d_{50}$ of the two main sands involved is very large. Similar conclusions were established in our earlier study on grain compaction (Mahmoodlu et al., 2016).

\subsection{Porosity and intrinsic permeability}

At the pore scale, porosity represents the ratio of the total volume of
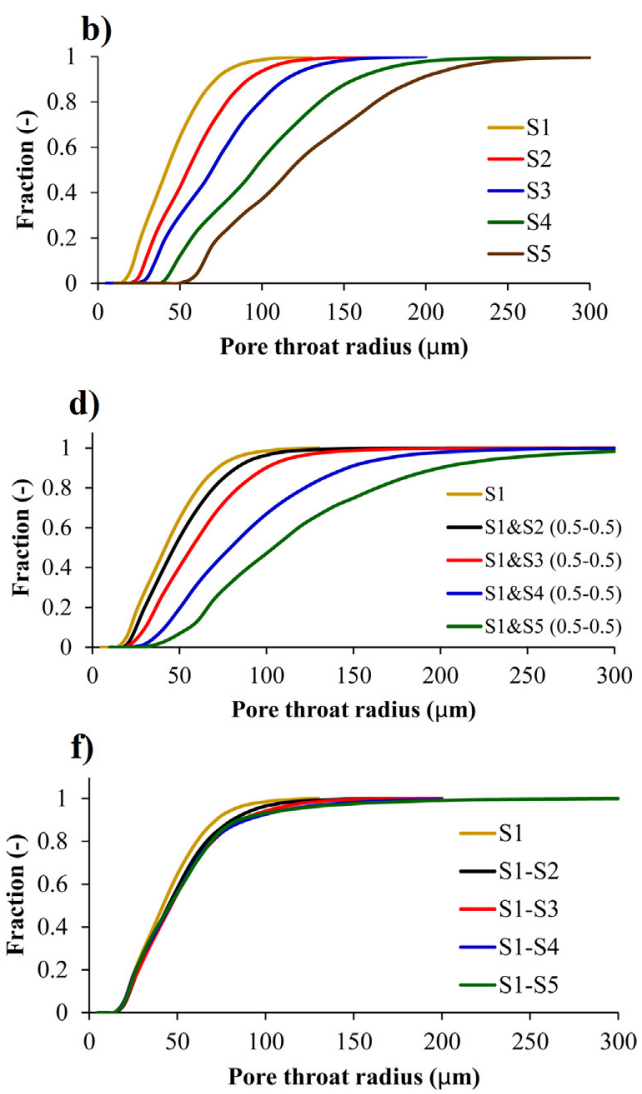

Fig. 4. Cumulative pore body and throat radii distributions for the five main sands (a and b), their mixtures with S1 (c and d), and the layered soil packings (e and f). Each mixture was obtained by mixing $50 \%$ of sand $\mathrm{S} 1$ with $50 \%$ of one of the other sands. All simulations had a target porosity of 0.35 . 

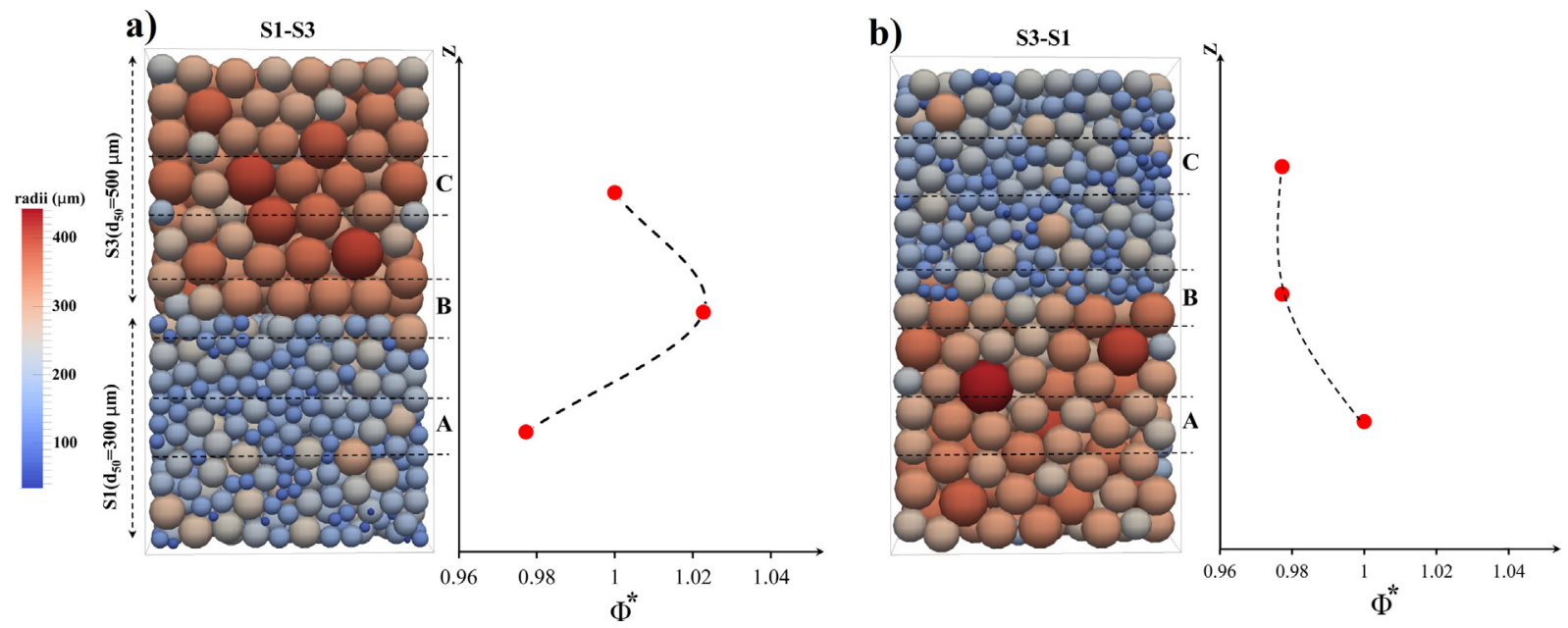

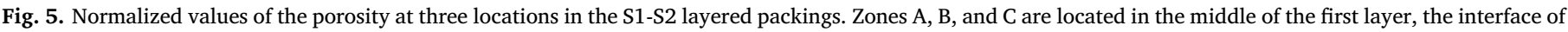

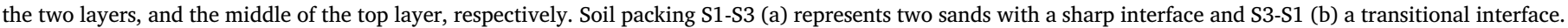
$\phi^{*}$ is the normalized porosity (porosity of each zone divided to the bulk porosity).

pore bodies and pore throats to the total volume of the porous medium. Hence, changes in porosity of a soil profile are directly affected by the changes in the pore sizes of the soil. Fig. 5 depicts changes in the porosity of a layered sand pack within three different zones (A, B, and C) along the sample profile. As expected, the porosity was found to increase at or near a sharp interface (Fig. 5a) as compared to the porosities of the two main sands individually (i.e., S1 and S3 in this case). However, notice that the porosity at the transitional interface (Fig. 5b) remained the same as that of the finer sand (here Sand S1). This we believe is due to the infiltration of small particles into the larger pores of the underlaying coarser layer, leading to smaller average pore sizes at the interface.

Soil permeability is determined by both the bulk geometric properties of a sample (e.g., porosity and the pore size distribution), as well as its topological properties such as pore space connectivity and tortuosity. We investigated the effects of pore structure on the permeability of each individual sand (i.e., Sands S1 to S5), of the mixtures of sand S1 (at a 0.5 fraction) with the other sands (S2 to S5), as well as of the layered soil packings. Results for the individual sands (Fig. 6a) show that the permeability of the main sands (S1 to S5) increased considerably and nonlinearly towards the coarsest sand with its largest $d_{50}$ value (i.e., sand $S 5$ with a $d_{50}$ of $885 \mu \mathrm{m}$ ). This confirms that the permeability is strongly affected by pore size (Håkansson and Lipiec, 2000; Assouline, 2006, Mahmoodlu et al., 2016).

To investigate the effect of grain heterogeneity on the permeability, sand S1 was mixed with the other sands (S2, S3, S4 and S4). For all calculations, the ratio of mixing was kept at $50 \%$ of $S 1$ and $50 \%$ of the other sands. As shown in Fig. 6d, mixing S1 with a coarser sand (S2 to S5) caused an increase in the fraction of smaller pores of the coarser sand and consequently a reduction in the permeability of sand S3. By contrast, the permeability of S2 increased due to an increase in the fraction of larger pores. We found that mixing sand S1 (having a $d_{50}$ of $283 \mu \mathrm{m}$ ) with S2 (having a $d_{50}$ of $385 \mu \mathrm{m}$ ) caused a small increase in the permeability due to small differences in $d_{50}$ values of the two sands, while mixing the coarser sands (S3, S4, and S5 with $d_{50}$ values of 485, 685 and $885 \mu \mathrm{m}$, respectively) increased the intrinsic permeability substantially. Our results clearly show that the permeability reductions of the coarser sand are dominated very much by the relative amounts of the finer sands (and hence the smaller pore sizes).

Changes in the bulk permeability, as a function $d_{50}$, of the layered sand packs having a sharp interface did not show a clear pattern (Fig. 6h). This may be because of the increased fraction of S1 in the layered sands when coarser sands were used. As noted earlier, the fraction of S1 varied from 70\% for packing S1-S2 to 95\% for packing
S1-S5 in order to keep the same thickness of each layer. However, the bulk permeability of a layered pack soil increased towards the coarse sand.

The relationships between permeability and pore size of the five main sands became slightly nonlinear towards coarse sand, as well as for the mixtures of $\mathrm{S} 1$ with the other sands (Fig. 6b, c, e, f, i, g). The relationships between permeability and pore size were even more nonlinear when the permeability at a given porosity of 0.35 is plotted as a function of either pore body radius or pore throat radius, as shown by the results in Fig. 6b, c, e, f, i, g. Although, the permeability versus pore body or throat radii showed nonlinear behavior for the sand with an interface, the plots suggest linear slopes which changes after the second (S1-S3) packing (Fig. 6i, g).

The effect of an interface on the bulk permeability of the layered sand samples was also investigated. Fig. 1S (Supplementary material) shows that a sharp interface causes an increase in the bulk permeability of the sand packing. This difference became more visible for the packings of S1 with sand having larger $d_{50}$ values. Fig. 7a depicts changes in the ratio of the bulk permeability to the average permeability of two sands, versus the ratio of $d_{50}$ of sand $S 1$ (when at the bottom of the sample) over $d_{50}$ of the second sand (S2 to S5 at the top). Results show that the ratio of the bulk permeability to average permeability is higher for the packings having a sharp interface (Fig. 7a). This ratio diminished for the packing of sand S1 with a coarser sand. In contrast with the interface effect on the bulk permeability, the permeability of mixtures of two sands increased for the packing of S1 with a coarser sand (Fig. 7b).

\subsection{Soil water retention curve}

This section shows how sample heterogeneity affects the soil water retention curve (SWRC), which has a first-order effect on predictions of water flow in variably saturated media. We investigated the effect of pore body and pore throat size as well as of the type of interface (sharp or transitional) between two sands in the layered samples on the SWRC.

We first studied the effect of grain size distribution on the SWRC. Fig. 8 depicts the evolution of water saturation, $S^{w}$ (given by $\theta^{w} / \theta_{s}^{w}$ ) as a function of capillary pressure, $P_{c}$, for the five main sands. Results indicate that the capillary pressure at a given saturation was always higher for the finer sands due to the creation of smaller pore throats regulating higher capillary entry pressures and controlling capillary displacement (see S1 in Fig. 8).

For the heterogeneity in terms of the grain size distribution simulations, combining S1 (fraction of 0.5 ) with a coarser sand (fraction of 
a)

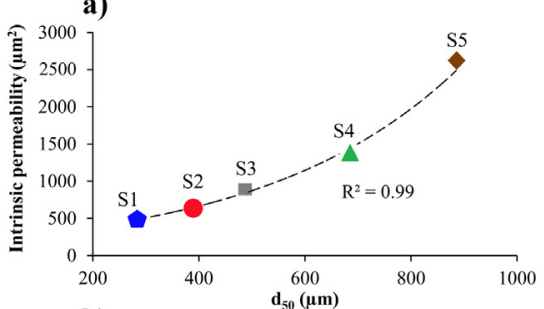

b)

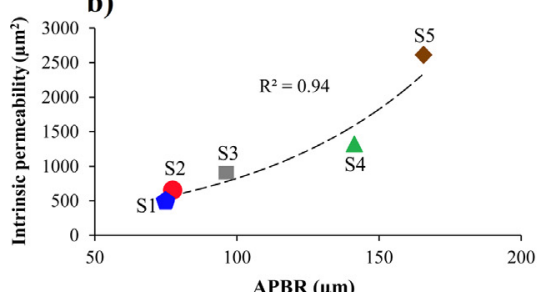

c)

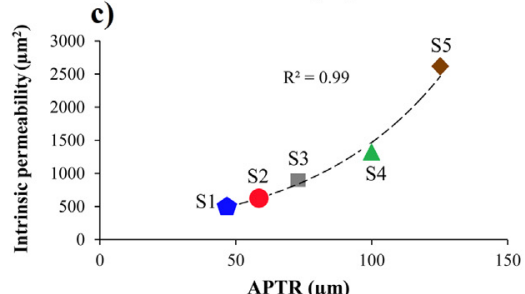

d)

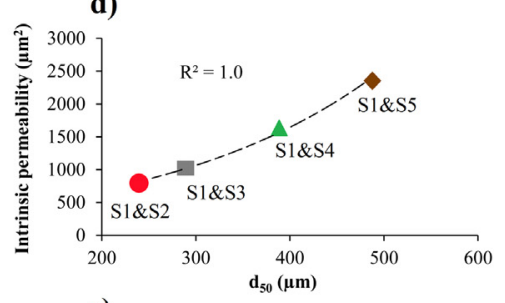

e)

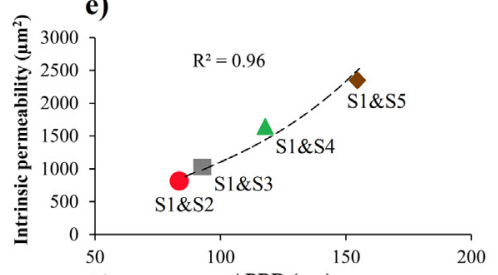

f )

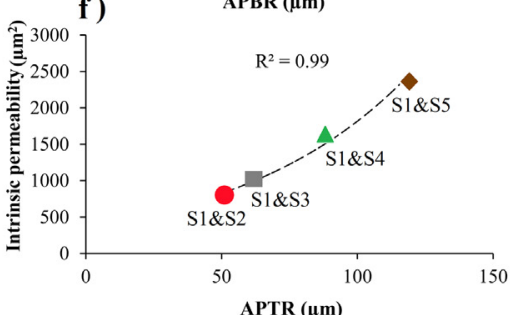

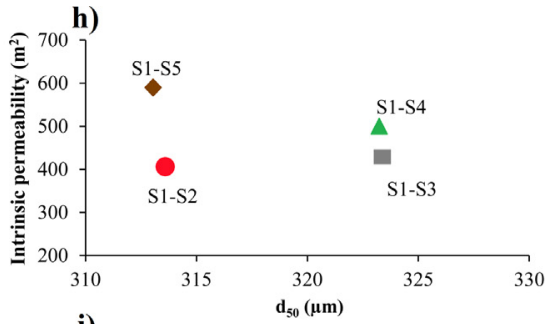

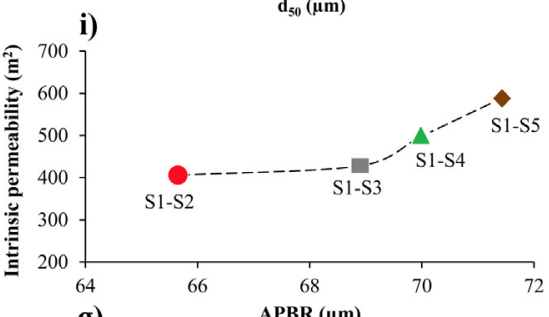

g)

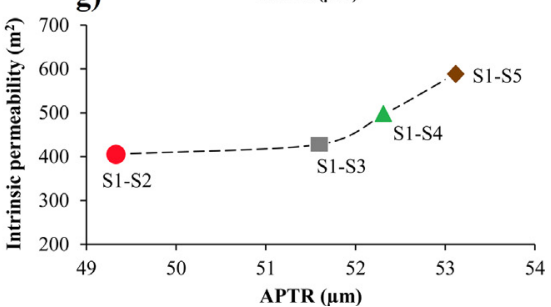

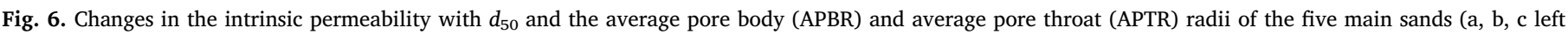

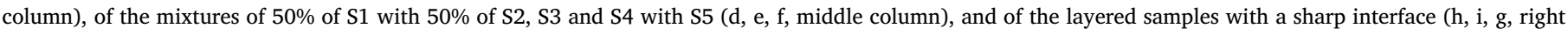
column).

0.5) caused a decrease in the capillary pressure at a given volumetric water content. This hence produced larger values of the van Genuchten $\alpha$ parameter in Eq. (3), and hence lower entry pressures (Fig. 9). The SWRCs of the combination of sand S1 with the other sands changed more non-uniformly towards the coarser sands. This is because of the creation of larger pore throat radii and their controlling effect on the SWRC of the mixture of S1 with coarser sand.

We next investigated the effects of sharp versus transitional interfaces on the SWRCs (Fig. 10). Calculated SWRCs of the layered sand packings with a sharp interface showed a clear effect of the interface when $d_{50}$ of two sands differed substantially from each other. By comparison, the SWRC of the sample with S1 (at the bottom) and S2 (at the top) showed a more monotonic change and did not exhibit a clear discontinuity at the interface of the two sands (Fig. 10). This is because the capillary pressures at which the water-filled pores start to drain are directly proportional to the equivalent radii of the pore throat (e.g., Durner, 1994). The radii of the throats for sands S1 and S2 were fairly close to each other, which leads to a monotonic change in the SWRC. Since the radii of the pore throats of S1 and the other of sands (e.g., S3, S4, and S5) were not in a same range, their mixtures should show a

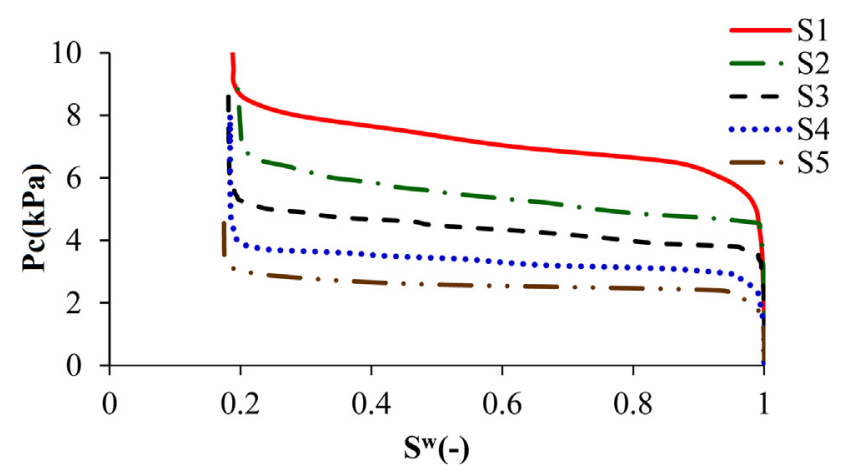

Fig. 8. Soil water retention curves of the five main sands.

more definite jump at the interface and less smooth behavior of the SWRCs.

As shown in Fig. 10, the drainage curves of the soil samples with a transitional interface (coarse sand at the bottom) showed relatively high residual water saturations, which increased for a layered sand
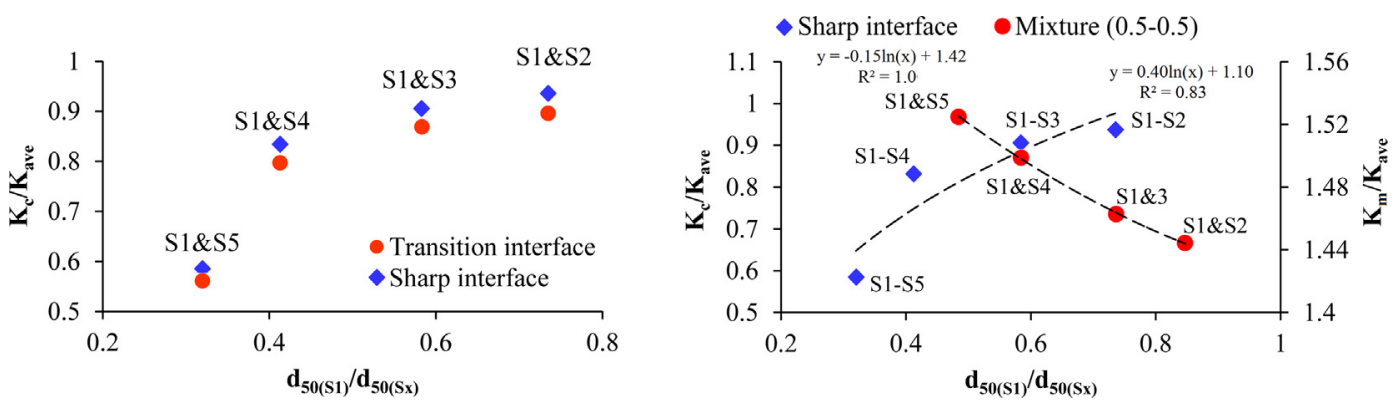

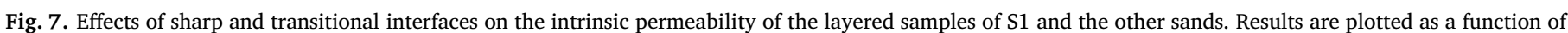

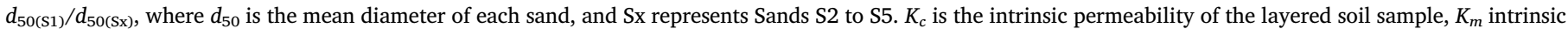

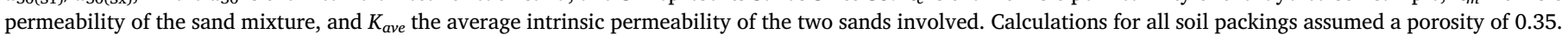


a)

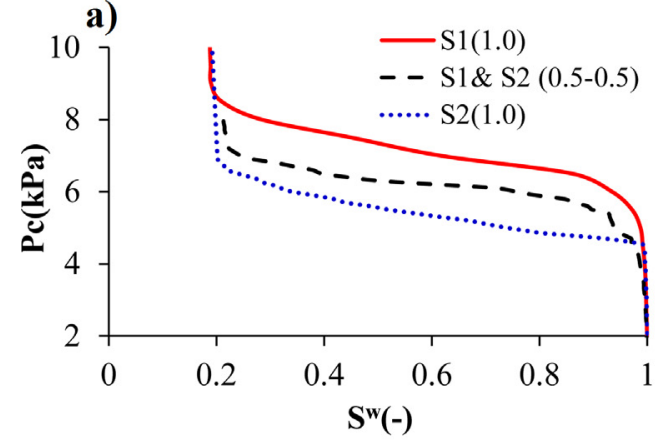

c)

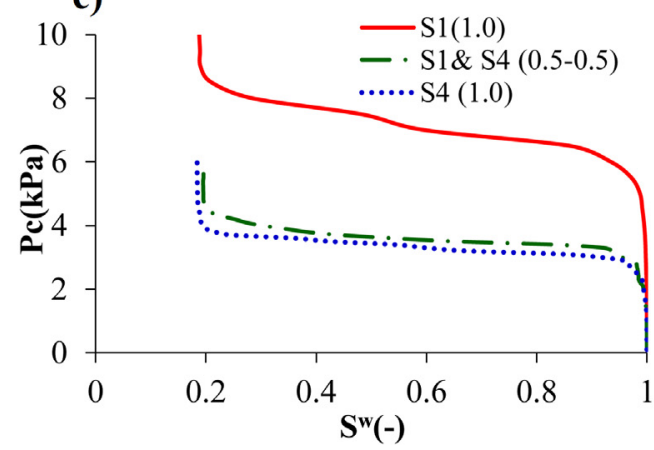

b)

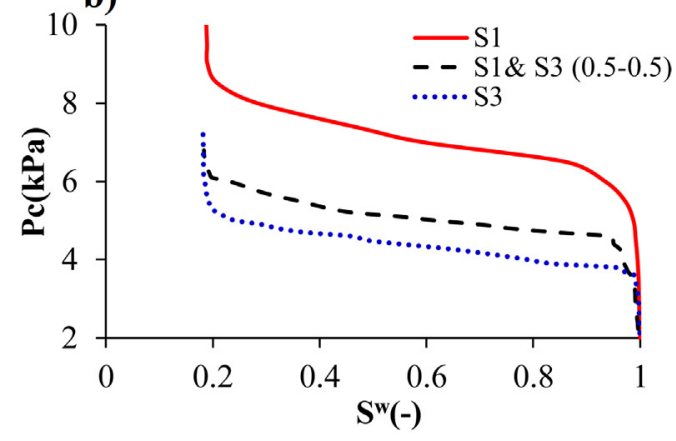

d)

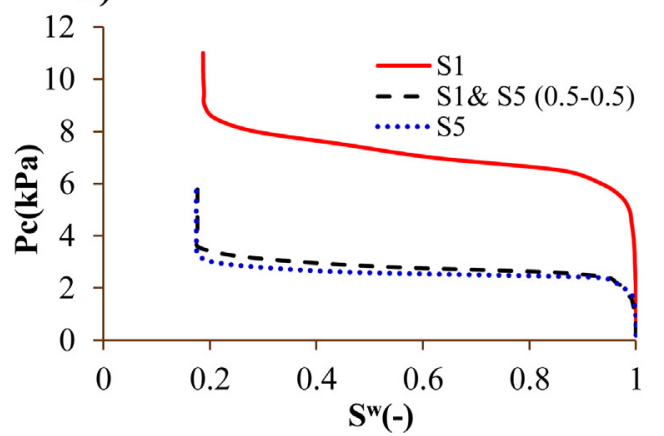

Fig. 9. Effect of mixing on the soil water retention curve.

pack having coarser sand (S5) at the bottom. Moreover, the SWRCs of the layered sands with a sharp interface showed very high van Genuchten $n$ values and residual water saturations (Table 2). In our simulations, air always invaded the soil sample from the top boundary. A possible explanation for the high residual saturations could be the high capillary pressure needed to drain the finer layer at the top (here S1). However, as soon as an air pathway reaches the interface, air will move into the coarser sand and thus bypass several upstream saturated pores. This may result in a constant, but non-uniform water saturation along
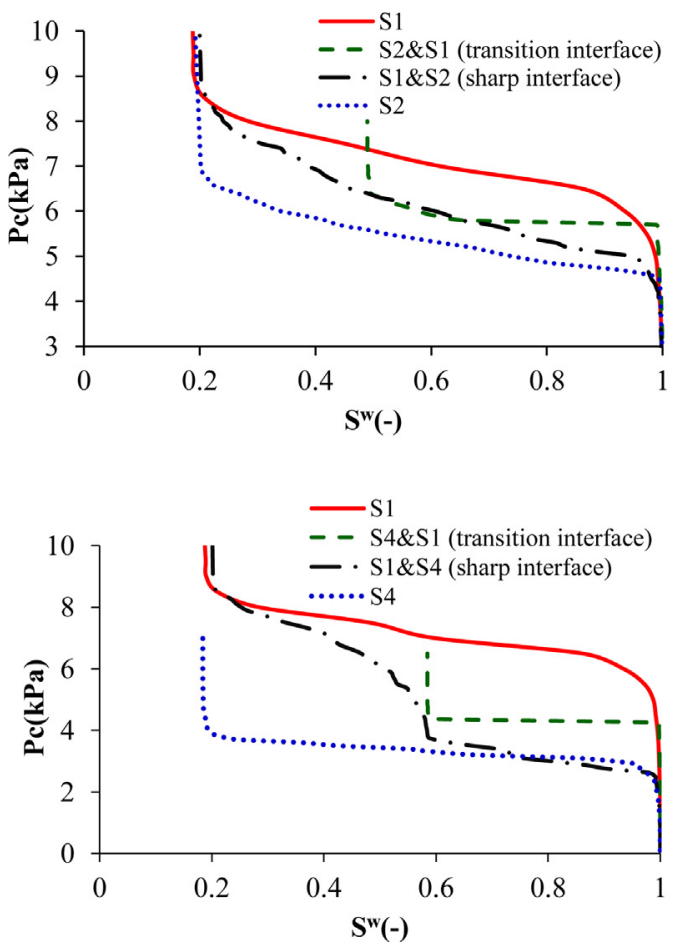

the sample.

Results of both main sands and their mixtures with S1 showed an increase in the van Genuchten $\alpha$ value towards sand having a larger value of $d_{50}$ (Table 2). The ratio of the van Genuchten parameter $\alpha$ of the sand mixture $\left(\alpha_{\text {mix }}\right)$ to the averages value of $\alpha\left(\alpha_{\text {ave }}\right)$ is plotted in Fig. 11a versus the ratio of $d_{50}$ of $S 1$ to $d_{50}$ of other sands. The plot shows a linear increase in the ratio of $\alpha_{\text {mix }}$ to $\alpha_{\text {ave }}$ when S1 is mixed with coarser material (i.e., the ratio of the $d_{50}$ values of the two sands).

Fig. $11 \mathrm{~b}, \mathrm{c}$ further depicts for the sharp interfaces the ratio of the
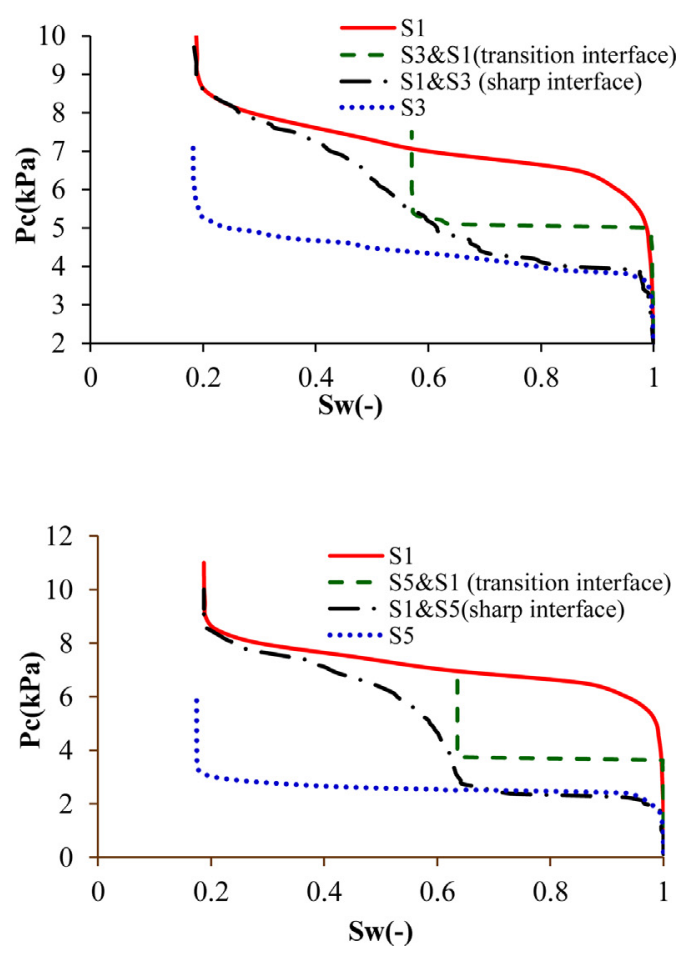

Fig. 10. Effect of sharp and transition interfaces on the soil water retention curve. 
a)

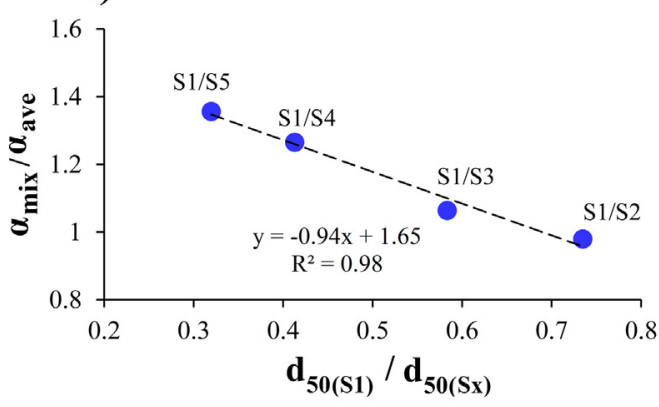

c)

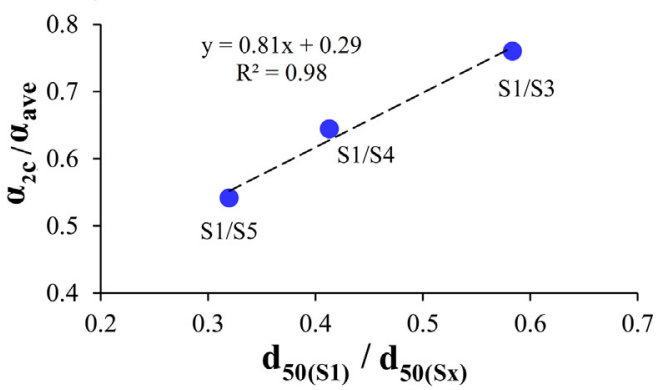

b)

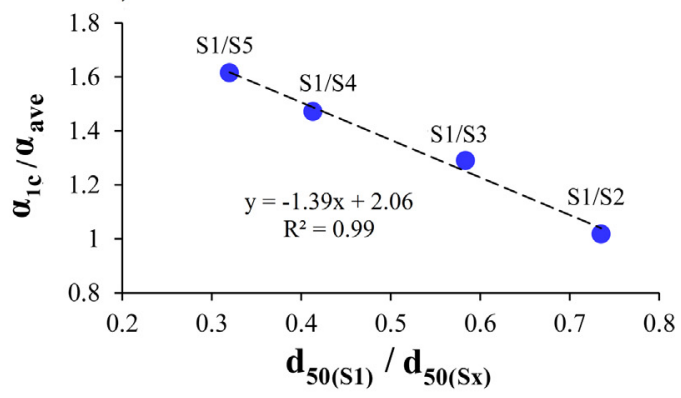

d)

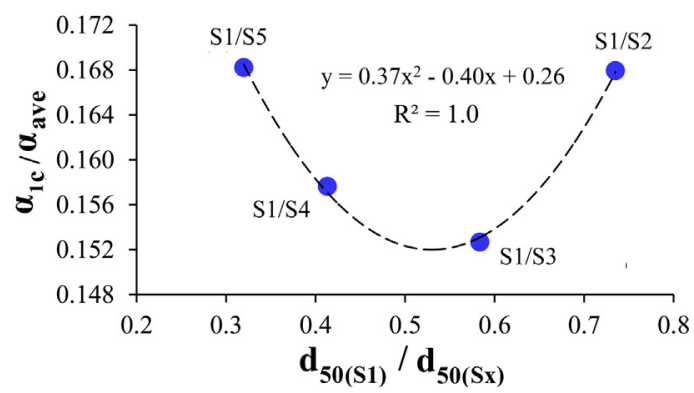

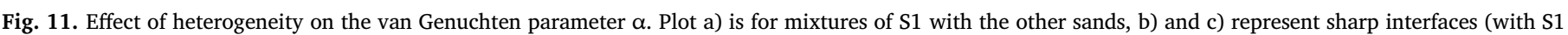

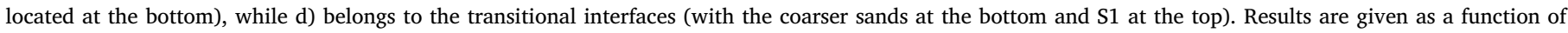
$d_{50(\mathrm{~S} 1)} / d_{50(\mathrm{Sx})}$, where $d_{50}$ is the mean diameter of each sand, and Sx represents Sands S2 to S5. Calculations for all soil packings assumed a porosity of 0.35 .

van Genuchten parameter $\alpha_{1}$ and $\alpha_{\text {ave }}$ versus the ratio of $d_{50}$ of $\mathrm{S} 1$ to $d_{50}$ of the other sands. We used $\alpha_{1}$ here for the coarser sand in the layered sample, while $\alpha_{a v e}$ is again the average of the $\alpha$ values of the two sands involved. Similarly as for mixing scenario, a linear relationship existed between the ratios of $\alpha_{1}$ to $\alpha_{a v e}$ versus the ratio of $d_{50}$ of S1 to $d_{50}$ of the other sands. However, we found a nonlinear relationship between the ratios $\alpha_{1} / \alpha_{\text {ave }}$ (for the layered-samples) to the ratio of $\alpha_{\text {mix }}$ to $\alpha_{\text {ave }}$ (for the sand mixtures), whereas the ratio $\alpha_{2} / \alpha_{\text {ave }}$ increased for two sands with a large ratio of $d_{50}$. In contrast with the sharp interfaces, the ratio $\alpha_{1} / \alpha_{\text {ave }}$ versus the ratio $\mathrm{d}_{50(\mathrm{~S} 1)} / \mathrm{d}_{50(\mathrm{Sx})}$ (where $S x$ denotes again S2 to S5) did not show linear behavior, but more of an inverse normal distribution.

\section{Conclusions}

In this study we used the discrete element method (DEM) to explore the effects of soil heterogeneity on pore structure and the unsaturated soil hydraulic properties (the intrinsic permeability and the soil water retention curve, SWRC) of five different sands and their mixtures. We found, as expected, that mixing of the finer sand with any of the coarse sands causes a reduction in the average pore body and pore throat sizes, and subsequently will diminish the intrinsic permeability values of the coarser sands.

For the layered porous media with a transitional interface, the local porosity of the soil decreased. This suggests that infiltration and sedimentation of small particles into larger pores of the coarser sands (typical of a graded bed in geological formations) decreases the soil pore sizes at the interface, and consequently reduces the local porosity of the soil at the interface. In contrast, and interestingly, the local porosity of the layered media with sharp interfaces increased due to deposition of larger grains on top of the fine grains. Results confirmed the development of a discontinuity between two layers through the creation of relatively large pores at the interface.

Non-linear relationships between permeability and the average pore body and throat radii were found when mixing different unimodal sands. One likely reason is variation in the soil pore sizes of the mixture of two unimodal sands. In general, the ratio of bulk permeability to the average permeability of two sands used in a two-layered packing increased more for the packings having a sharp interface.

Calculated SWRCs showed that mixtures of the finer sand with a coarser sand caused a decrease in the capillary pressure of the coarser sand. This in turn produced larger values of the van Genuchten parameter $\alpha$ and hence lower entry pressures. We found the ratio of the $\alpha$ (of the mixtures) to the average value of $\alpha$ to increase with larger differences in the $d_{50}$ values of the two sands involved.

Sharp interfaces can cause a non-monotonic change in the drainage curve due to discrepancies between the pore throat radii of the two sands in the sample. This change is influenced by $d_{50}$ or the grain size distribution of the sands used in the two-layered packing. In contrast with sharp interfaces, transitional interfaces produced monotonic changes in the drainage curves for two sands having a small difference in their $d_{50}$ values.

High residual water saturations were found for the drainage curves of sand samples having a transitional interface (with coarse sand at the bottom). Residual saturations increased with the $d_{50}$ value of the sand at the bottom of the sample. Similarly as for the mixture scenario, the ratio of the van Genuchten parameter $\alpha$ to the average value of $\alpha$ of the two sands involved, increased with larger difference in the $d_{50}$ values of the sands.

Finally, we note that our study involved artificially created porous media made up of spherical particles. The results give much insight in how particle mixing and layering affect the unsaturated soil hydraulic properties. We acknowledge that comparisons with actual experimental data are very much needed to more definitely confirm our findings.

\section{Acknowledgments}

The authors would like to thank Hamed Aslannejad (Utrecht University) for his technical assistance. Comments by four anonymous referees and the Associate Editor of Journal of Hydrology further helped to improve the manuscript. 


\section{Appendix A. Supplementary data}

Supplementary data associated with this article can be found, in the online version, at https://doi.org/10.1016/j.jhydrol.2018.07.060.

\section{References}

Al-Ismaily, S.S., Al-Maktoumi, A.K., Kacimov, A.R., Al-Saqri, S.M., Al-Busaidi, H.A., 2015. Impact of a recharge dam on the hydropedology of arid zone soils in Oman: anthropogenic formation factor. J. Hydrol. Eng. 20 (4). https://doi.org/10.1061/ (ASCE)HE.1943-5584.0000886.

Alonso, E.E., Cardoso, R., 2010. Behavior of materials for earth and rockfill dams: perspective from unsaturated soil mechanics. Front. Archit. Civil Eng. China 4 (1), 1-39.

Antinoro, C., Arnone, E., Noto, L.V., 2017. The use of soil water retention curve models in analyzing slope stability in differently structured soils. Catena 150, 133-145.

Arora, B., 2012. Modeling Biogeochemistry and Flow within Heterogeneous Formations in Variably-Saturated Media. Doctoral Dissertation. A\&M University, Texas.

Assouline, S., 2006. Modeling the relationship between soil bulk density and the hydraulic conductivity function. Vadose Zone J. 5, 697-705.

Belheine, N., Plassiard, J.P., Donzé, F.V., Darve, F., Seridi, A., 2009. Numerical simulation of drained triaxial test using 3D discrete element modeling. Comput. Geosci. 36, 320-331.

Bittelli, M., Flury, M., 2009. Errors in water retention curves determined with pressure plates. Soil Sci. Soc. Am. J. 73, 1453-1460.

Chareyre, B., Cortis, A., Catalano, E., Barthélemy, E., 2012. Pore-scale modeling of viscous flow and induced forces in dense sphere packings. Transport Porous Med. 94 (2), 595-615.

Chen, P.Y., Chen, C.H., Hsu, N.S., Wu, C.M., Wen, J.C., 2012. Influence of heterogeneity on unsaturated hydraulic properties: (1) local heterogeneity and scale effect. Hydrol. Process. 26 (23), 3593-3603.

Cnudde, V., Boone, M.N., 2013. High-resolution X-ray computed tomography in geosciences: a review of the current technology and applications. Earth Sci. Rev. 123, 1-17. https://doi.org/10.1016/j.earscirev.2013.04.003.

Coppola, A., Basile, A., Comegna, A., Lamaddalena, N., 2009. Monte Carlo analysis of field water flow comparing uni-and bimodal effective hydraulic parameters for structured soil. J. Contam. Hydrol. 104 (1), 153-165.

Cundall, P.A., Strack, O.D.L., 1979. A discrete numerical model for granular assemblies. Geotechnique 29, 47-65. https://doi.org/10.1680/geot.1979.29.1.47.

Dane, J.H., Hopmans, J.W., 2002. Hanging water column. p. 680-684. In: J.H. Dane and G.C. Topp (ed.) Methods of soil analysis. Part 4. SSSA Book Ser. 5. SSSA, Madison, WI.

Di Prima, S., Bagarello, V., Bautista, I., Burguet, M., Cerdà, A., Iovino, M., Prosdocimi, M., 2016. Comparing a simple methodology to evaluate hydrodynamic parameters with rainfall simulation experiments. EGU General Assembly 2016, held 17-22 April, 2016 in Vienna Austria, p.289.

Dubois, V., Abriak, N.E., Zentar, R., Ballivy, G., 2009. The use of marine sediments as a pavement base material. Waste Manage. 29 (2), 774-782.

Durner, W., 1994. Hydraulic conductivity estimation for soils with heterogeneous pore structure. Water Resour. Res. 30 (2), 211-223.

Falode, O., Manuel, E., 2014. Wettability effects on capillary pressure, relative permeability, and irredcucible saturation using porous plate. J. Pet. Eng. ID 465418, 12p. https://doi.org/10.1155/2014/465418.

Gee, G., Or, D., 2002. Particle size analysis. In: J. J. Dane and G. C. Topp (eds.), Methods of Soil Analysis, Part 4. Physical Methods, 255-294, Soil Sci. Soc. Am., Madison, WI.

Gladkikh, M., Bryant, S., 2005. Prediction of imbibition in unconsolidated granular materials. J. Colloid Interface Sci. 288, 526-539.

Håkansson, I., Lipiec, J., 2000. A review of the usefulness of relative bulk density values in studies of soil structure and compaction. Soil Till. Res. 53 (2), 71-85.

Hohenbrink, T.L., Lischeid, G., 2015. Does textural heterogeneity matter? quantifying transformation of hydrological signals in soils. J. Hydrol. 523, 725-738.

Huang, M., Lee Barbour, S.L., Elshorbagy, A., Zettl, J.D., Cheng Si, B., 2011. Infiltration and drainage processes in multi-layered coarse soils. Can. J. Soil Sci. 91 (2), 169-183. https://doi.org/10.4141/CJSS09118.

Katukiza, A.Y., Ronteltap, M., Niwagaba, C.B., Kansiime, F., Lens, P.N.L., 2014. Grey water treatment in urban slums by a filtration system: optimisation of the filtration medium. J. Environ. Manage. 146, 131-141.

Keller, T., Lamandé, M., Peth, S., Berli, M., Delenne, J.Y., Baumgarten, W., Rabbel, W., Radjai, F., Rajchenbach, J., Selvadurai, A.P.S., Or, D., 2013. An interdisciplinary approach towards improved understanding of soil deformation during compaction. Soil Till. Res. 128, 61-80.

Lopes, R.L., Jucá, J.F.T., Mariano, M.O.H., 2013. Water infiltration in final cover layer of landfills in northeast region of Brazil. Adv. Unsaturated Soils. CRC Press 243-248. https://doi.org/10.1201/b14393-33.

Mahmoodlu, M.G., Raoof, A., Sweijen, T., van Genuchten, M.T., 2016. Effects of sand compaction and mixing on pore structure and the unsaturated soil hydraulic properties. Vadose Zone J. 15 (8). https://doi.org/10.2136/vzj2015.10.0136.

Mehlhorn, T., Prohaska, S., Homberg, U., Slowik, V., 2008. Modelling and analysis of particle and pore structures in soils. In: Witt, K.J. (Ed.), Workshop Internal Erosion.
26-28 Nov. 2008. Schriftenreihe Geotechnik 21. Bauhaus-Universität, Weimar, Germany, pp. 53-60.

Mohanty, B.P., Zhu, J., 2007. Effective hydraulic parameters in horizontally and vertically heterogeneous soils for steady-state land-atmosphere interaction. J. Hydrometeorol. 8 (4), 715-729.

Morbidelli, R., Saltalippi, C., Flammini, A., Corradini, C., Brocca, L., Govindaraju, R.S., 2016. An investigation of the effects of spatial heterogeneity of initial soil moisture content on surface runoff simulation at a small watershed scale. J. Hydrol. 539, 589-598.

Mousavi, M.A., Bryant, S.L., 2012. Connectivity of pore space as a control on two-phase flow properties of tight-gas sandstones. Transport Porous Med. 94 (2), 537-554. https://doi.org/10.1007/s11242-012-0017-x.

Mukhlisin, M., Baidillah, M.R., Taha, M.R., El-Shafie, A., 2011. Effect of soil water retention model on slope stability analysis. Int. J. Phys. Sci. 6 (19), 4629-4635.

Nimmo, J.R., Perkins, K.S., Lewis, A.M., 2002. Steady-State Centrifuge, Methods of Soil Analysis. In: Inc, S.S.S.A. (Ed.), Part 4. Madison, WI, pp. 903-916.

Perrier, E., Rieu, M., Sposito, G., Marsily, G., 1996. Models of the water retention curve for soils with a fractal pore size distribution. Water Resour. Res. 32 (10), 3025-3031.

Peth, S., Nellesen, J., Fischer, G., Horn, R., 2010. Non-invasive 3D analysis of local soil deformation under mechanical and hydraulic stresses by mCT and digital image correlation. Soil Tillage Res. 111, 3-18. https://doi.org/10.1016/j.still.2010.02.007.

Prodanović, M., Mehmani, A., Sheppard, A.P., 2015. Imaged-based multiscale network modelling of microporosity in carbonates. Geological Society, London, Special Publications 406 (1), 95-113.

Purushothama, R.P., 2007. Soil Mechanics and Foundation Engineering, Second edition. Pearson Education India, 840 pages.

Raoof, A., Hassanizadeh, S.M., 2012. A new formulation for pore-network modeling of two-phase flow. Water Resour. Res. 48 (1), 1-13.

Raoof, A.S.M., Hassanizadeh, S.M., Leijnse, A., 2010. Upscaling transport of adsorbing solutes in porous media: pore-network modeling. Vadose Zone J. 9 (3), 624-636. https://doi.org/10.2136/vzj2010.0026.

Sakaki, T., Smits, K.M., 2015. Water retention characteristics and pore structure of binary mixtures. Vadose Zone J. 14 (2). https://doi.org/10.2136/vzj2014.06.0065.

Scanlon, B.R., Andrasky, B.J., Bilskie, J., 2002. Miscellaneous methods for measuring matric or water potential. In: Dane, J.H., Topp, G.C. (Eds.), Methods of soil analysis. Part 4. Physical methods. SSSA Book Ser. 5. SSSA, Madison, WI, pp. 643-670.

Šmilauer, V., Catalano, E., Chareyre, B., Dorofeenko, S., Duriez, J., Gladky, A., Kozicki, J., Modenese, C., Scholtès, L., Sibille, L., Stránský, J., Thoeni. K., 2015. Yade Documentation (V.Šmilauer, ed.), The Yade Project, 1st ed.

Sweijen, T., Nikooee, E., Hassanizadeh, S.M., Chareyre, B., 2016. The effects of swelling and porosity change on capillarity: DEM coupled with a pore unit assembly method. Transport Porous Med. 113 (1), 207-226.

Sweijen, T., Aslannejad, H., Hassanizadeh, S.M., 2017. Capillary pressure-saturation relationships for porous granular materials: pore morphology method vs. pore unit assembly method. Adv. Water Resour. 107, 22-31.

Tong, A.T., Catalano, E., Chareyre, B., 2012. Pore-scale flow simulations: model predictions compared with experiments on bi-dispersed granular assemblies. Oil Gas Sci. Technol. 67, 743-752. https://doi.org/10.2516/ogst/2012032.

Torskaya, T., Shabro, V., Torres-Verdín, C., Salazar-Tio, R., Revil, A., 2014. Grain shape effects on permeability, formation factor, and capillary pressure from pore-scale modeling. Transport Porous Med. 102 (1), 71-90.

Unsal, E., Mason, G., Morrow, N.R., Ruth, D.W., 2009. Bubble snap-off and capillary back pressure during counter-current spontaneous imbibition into model pores. Langmuir $25,3387-3395$.

van Genuchten, M.Th., 1980. A closed-form equation for predicting the hydraulic conductivity of unsaturated soils. Soil Sci. Soc. Am. J. 44 (5), 892-898.

van Genuchten, M.Th., Leij, F.J., Yates, S.R., 1991. The RETC code for quantifying the hydraulic functions of unsaturated soils. Report No. EPA/600/2-91/065. R.S. Ker Environmental Research Laboratory, U. S. Environmental Protection Agency, Ada, OK. 85 p. https://www.pc-progress.com/en/Default.aspx?retc).

Vasilyev, L., Raoof, A., Nordbotten, J.M., 2012. Effect of mean network coordination number on dispersivity characteristics. Transport Porous Med. 95 (2), 447-463.

Vaz, C.M.P., Tuller, M., Lasso, P.R.O., Crestana, S., 2014. New perspectives for the application of high-resolution benchtop X-ray microCT for quantifying void, solid and liquid phases in soils. In: W.G. Teixeira et al., editors, Application of soil physics in environmental analyses: Measuring, modelling and data integration. Progr. Soil Sci. Springer Int. Publ., Cham, Switzerland. 261-281. doi:10.1007/978-3-319-060132 212.

Yuan, C., Chareyre, B., Darve, F., 2015. Pore-scale simulations of drainage in granular materials: finite size effects and the representative elementary volume. Adv. Water Resour. 95, 109-124.

Zhang, Q., Raoof, A., Hassanizadeh, S.M., 2015. Pore-scale study of flow rate on colloid attachment and remobilization in a saturated micromodel. J. Environ. Qual. 44 (5), 1376-1383.

Zhu, J., Sun, D., 2012. Soil hydraulic properties for moisture redistribution in a largescale heterogeneous landscape. Hydrol. Sci. J. 57 (6), 1196-1206.

Zhuang, L., Hassanizadeh, S.M., Qin, C.Z., de Waal, A., 2017. Experimental investigation of hysteretic dynamic capillarity effect in unsaturated flow. Water Resour. Res. 53 (11), 9078-9088. 